\title{
Open multi-agent systems with discrete states and stochastic interactions
}

\author{
Vineeth S Varma*, Irinel-Constantin Morărescu*, Dragan Nešić\#
}

\begin{abstract}
We consider a multi-agent system in which agents arrive and depart from a network randomly as a Bernoulli process. Each agent that is active in the network must decide between two actions represented by 0 or 1 . Each active agent then observes the action of a random neighbour and updates its preference towards a certain action. New agents that arrive into the network are activated with a random preference and action. This means that the notion of consensus in the standard sense can no longer be applied and instead, we provide conditions under which majority action preservation occurs when the number of agents is arbitrarily large. This property will imply that a large fraction of the active agent population will retain their action almost surely.
\end{abstract}

Index Terms-Multi-agent systems, Markov chains, open systems, consensus.

\section{INTRODUCTION}

The analysis of multi-agent systems received an increasing attention during the last decades. They have a wide range of applications covering robotics [1], [2], power networks [3], [4] and opinion dynamics [5], [6]. In the framework of multi-agent systems, the most studied problem is the consensus achievement [7], [8], [9], which captures various coordination behaviors.

Some of the most cited features leading to the usage of multi-agent systems are their flexibility and scalability. While the multi-agent systems literature mainly treats the case of fixed dimension networks, the size of physical networks can increase or decrease in time. Indeed, a typical example is the one of a social network whose size is time-varying and often very large but it does not increase unbounded. On the other hand, technological networks may also suffer from failure/repairing of some agents leading to modifications of the active subsystems in the network. This motivates us to analyze here the behavior of open multi-agent systems, in which agents keep arriving and/or leaving the network during the evolution in time.

Open multi-agent systems have been empirically studied in [10], [11] in the framework of opinion dynamics. In computer science, there exist specific architectures coping with the possibility of agents joining and leaving the system [12]. A formal mathematical analysis of open multi-agent systems with deterministic arrivals and departures has been recently provided in [13], in which, all the dynamics are

This work was partially supported by the Australian Research Council under the Discovery Project SP170104099 and the French CNRS PEPS project YPSOC.

* Université de Lorraine, CNRS, CRAN, F-54000 Nancy, France vineeth. satheeskumar-varma@univ-lorraine.fr, constantin.morarescu@univ-lorraine.fr

\# Department of Electrical and Electronic Engineering, The University of Melbourne, Parkville, 3010 VIC, Australia. dnesic@unimelb. edu.au deterministic, and agents are only allowed to arrive, and do not depart.

As for [13], [14], a precise motivation of the present work is given by opinion dynamics in social networks. Instead of imposing pre-determined or periodic time instants for arrivals like [13], we assume that arrivals and departures occur with certain probabilities. Another important difference is that we consider random gossiping over the time-varying complete graph of active agents, with each agent having access only to a quantized version of the state of random neighbours, and not to the exact value. An important idea that we introduce in this paper, which is also highly relevant for opinion dynamics, is the one of almost sure consensus. This also represents a major difference with respect to [14] in which we study a closed system (i.e., the number of agents is fixed) that always leads to consensus. We note that gossiping under quantized communication for closed systems has been studied in [15]. Beside the fact that we consider open systems, in our model, the state of any agent can evolve only inside a discrete/finite set of values which seems more appropriate for many opinion dynamics applications.

The main challenges that we face in this work are the timevarying dimension of the system and the absence of classical "convergence". To overcome the first challenge, we consider that there exists a fixed, but arbitrarily large dimension of the system denoted by $N$. Consequently, at any instant of time $t$, only a fraction of the $N$ agents are active, and their number characterizes the real size of the network, while the others are inactive and artificially added to facilitate the analysis. We assign a precise state value to active agents that form the network at time $t$ while inactive agents have a generic inactive state referred to as NA.

The second challenge is that consensus in the classical sense is impossible to achieve due to repeated arrivals with a different state, and this is indeed one reason why not many works have theoretically studied consensus in open systems. Therefore, we propose and analyze a different notion of consensus, which requires that a large fraction of the active population maintains a common action (i.e., the quantized version of the state) almost surely. The main objective of this work is to provide conditions under which, the proposed dynamics stabilizes the consensus points almost surely. Our results are suitable for application to several scenarios where consensus of discrete actions are required such as synchronizing the wireless channel with which mobile devices communicate, opinion dynamics, etc.

The rest of the paper is organized as follows. Section II introduces the main concepts and formulates the problem under consideration. Section II-B mathematically formulates 
the system dynamics as a Markov chain. The main results concerning the equilibria of large-scale multi-agent systems under the proposed stochastic dynamics are presented in Section III. The results of our numerical studies and simulations are illustrated in Section IV. The paper ends with some concluding remarks and perspectives.

Notation. We use $\mathbb{R}$ for the set of real numbers, $\mathbb{R}_{+}$ the set of non-negative reals and $\mathbb{N}=\{0,1, \ldots\}$ the set of non-negative integers. The indicator function is given by $\mathbf{1}_{S}(x)$ which takes the value 1 when $x \in S$ and 0 otherwise. Throughout the paper the function $\lfloor x\rceil$ represents the integer part of $x+\frac{1}{2}$. The sign function which is 1 when $x>0,0$ if $x=0$ and -1 if $x<0$ is denoted by $\operatorname{sgn}(x)$. We also use $O(\cdot)$ for the big $O$ notation describing the limiting behavior. As our consensus dynamics has stochastic elements, we also introduce $\operatorname{Pr}(A)$ to denote the probability of event $A$, and $\mathbb{E}[\cdot]$ for the expectation of a random variable. The notation $\operatorname{Pr}(A \mid B)$ is used for the the probability of event $A$ conditioned by the event $B$. We also use almost surely to describe events that happen with probability 1 and define the corresponding stability notion for any stochastic process $p(t)$.

Definition 1 (Almost surely stable): If there exists $\epsilon *>0$ s.t.,

$$
\operatorname{Pr}\left(\left|p(t+1)-p^{*}\right|<\epsilon|| p(t)-p_{A}^{*} \mid<\epsilon\right)=1
$$

for all $\epsilon<\epsilon_{A}^{*}$, then we say that $p^{*}$ is almost surely stable for the stochastic dynamics of $p(t)$.

\section{Problem Statement}

\section{A. System dynamics}

We consider a discrete-time multi-agent system with $t \in \mathbb{N}$ denoting the time instants. We use $\mathcal{V}:=\{1,2, \ldots, N\}$ to denote the set of agents. At any given time instant $t$, only a subset $\mathcal{V}_{A}(t) \subseteq \mathcal{V}$ of agents are active in the network. We use $\mathcal{V}_{I}(t):=\mathcal{V} \backslash \mathcal{V}_{A}(t)$ to denote the set of inactive agents. Let us also introduce a fixed value $L \in 2 \mathbb{N}+1$ and the set $\mathcal{L}=\left\{l_{0}, l_{1}, \ldots, l_{L}\right\}$ with $l_{k}=\frac{k}{L}, \forall k \in\{0,1, \ldots, L\}$. When an agent is inactive, we assign the state NA, i.e. for all $i \in \mathcal{V}_{I}(t), x_{i}(t)=\mathrm{NA}$. We call the extended state space $\mathcal{L}_{E}=\mathcal{L} \cup\{\mathrm{NA}\}$.

We assign to any agent $i \in \mathcal{V}_{A}(t)$ a hidden state/preference $x_{i}(t) \in \mathcal{L}$ and a visible action based on its state as

$$
q_{i}(t)=\left\lfloor x_{i}(t)\right\rceil \text {. }
$$

It is worth noting that, when $L=1$, the state and the action sets are identical. Since we consider an open system, any agent which is active at time $t$ i.e., $i \in \mathcal{V}_{A}(t)$, may become inactive at $t+1$ with a probability $\delta \in(0,1)$. Similarly, any inactive agent $i \in \mathcal{V}_{I}(t)$ may activate at $t+1$ with a probability $\gamma$. This is:

$$
\begin{aligned}
& \operatorname{Pr}\left(x_{i}(t+1)=\mathrm{NA} \mid x_{i}(t) \in \mathcal{L}\right)=\delta, \\
& \operatorname{Pr}\left(x_{i}(t+1) \in \mathcal{L} \mid x_{i}(t)=\mathrm{NA}\right)=\gamma,
\end{aligned}
$$

On top of this, at any time $t$ such that $\left|\mathcal{V}_{A}(t)\right| \geq 2$ (i.e., more than one agent is active), an agent $i \in \mathcal{V}_{A}(t)$ may have only one neighbour with index $J_{i}(t)$ which is randomly chosen among all the other active agents:

$\operatorname{Pr}\left(J_{i}(t)=j\right)=\frac{1}{\left|\mathcal{V}_{A}(t)\right|-1}, \forall i \in \mathcal{V}_{A}(t), j \in \mathcal{V}_{A}(t) \backslash\{i\}$

Moreover, the agent $i \in \mathcal{V}_{A}(t)$ will update with probability $\beta \in(0,1-\delta)$ its state $x_{i}(t)$ by observing the action of its neighbour $J_{i}(t) \in \mathcal{V}_{A}(t) \backslash\{i\}$ as follows:

$$
\operatorname{Pr}\left(x_{i}(t+1)=\phi\left(x_{i}(t), q_{J_{i}(t)}(t)\right) \mid x_{i}(t) \in \mathcal{L}\right)=\beta
$$

where

$$
\phi(\ell, q)=\ell+\frac{1}{L} \operatorname{sgn}(q-\ell)
$$

for any $\ell \in \mathcal{L}, q \in\{0,1\}$. This model is inspired by [16] in which a Bayessian update rule was proposed in the context of opinion dynamics. Additionally, classical discrete-state multi-agent dynamics like the Ising model, which can be seen as a special case of (4) when $L=1$, do not lead to consensus in open systems as we show later in Remark 2.

In other words, the system dynamics is defined by random gossiping which indicates that any active agent can become a neighbour of $i$ with identical probabilities. Finally, the active agent may chose to retain its preference if the above events don't occur, i.e.,

$$
\begin{gathered}
\operatorname{Pr}\left(x_{i}(t+1)=x_{i}(t) \mid x_{i}(t) \in \mathcal{L}\right)=1-\delta-\beta, \\
\operatorname{Pr}\left(x_{i}(t+1)=x_{i}(t) \mid x_{i}(t)=\mathrm{NA}\right)=1-\gamma .
\end{gathered}
$$

When an inactive agent enters the network and becomes active, it must decide on a certain state. We consider that agents chose a random state with identical probabilities, i.e.,

$$
\operatorname{Pr}\left(x_{i}(t+1)=\ell \mid x_{i}(t)=\mathrm{NA}\right)=\frac{\gamma}{L+1}
$$

for any $\ell \in \mathcal{L}$. Note that any agent which becomes inactive does not retain any memory of its preference while it was active. We can use (2)-(7) to describe the proposed algorithm for achieving majority consensus in action/decision. When $\left|\mathcal{V}_{A}(t)\right| \geq 1$, define $p_{-}(t)$ as the fraction of the active population with action 0 , and $p_{+}(t)$ as the fraction with action 1 , calculated as

$$
p_{+}(t)=\frac{\sum_{n \in \mathcal{V}_{A}(t)} q_{n}(t)}{\left|\mathcal{V}_{A}(t)\right|}
$$

and $p_{-}(t)=1-p_{+}(t)$. Throughout the paper we also use $p_{A}(t)$ to denote the fraction of active agents, i.e.,

$$
p_{A}(t)=\frac{\left|\mathcal{V}_{A}(t)\right|}{N}=\frac{\sum_{n=1}^{N} \mathbf{1}_{\mathcal{L}}\left(x_{n}(t)\right)}{N}
$$

Next, at any $t$ such that $p_{A}(t)>0$, we define

$$
p_{\ell}(t)=\frac{\sum_{n=1}^{N} \mathbf{1}_{\{\ell\}}\left(x_{n}(t)\right)}{\sum_{n=1}^{N} \mathbf{1}_{\mathcal{L}}\left(x_{n}(t)\right)}
$$

for any $\ell \in \mathcal{L}$, which denotes the fraction of active agents with state $\ell$. Note that we can also write

$$
p_{+}(t)=\frac{\sum_{n \in \mathcal{V}_{A}(t)} q_{n}(t)}{\left|\mathcal{V}_{A}(t)\right|}=\sum_{k=\frac{L+1}{2 L}}^{L} p_{l_{k}}(t)
$$




\section{B. Markov model}

In order to give a complete mathematical model of the open system that takes into account all the stochastic phenomena associated with the activation/deactivation of agents as well as possibility of update or not, we formulate the dynamics as a Markov process in which $\forall i \in \mathcal{V}, \ell \in \mathcal{L}$ :

$$
\left\{\begin{array}{l}
\operatorname{Pr}\left(x_{i}(t+1)=\ell+\frac{\operatorname{sgn}(1-\ell)}{L} \mid x_{i}(t)=\ell \in \mathcal{L}\right) \\
=\beta \operatorname{Pr}\left(\left\lfloor x_{J_{i}(t)}(t)\right\rceil=1\right)=\beta \frac{\sum_{j \in \mathcal{V}_{A}(t) \backslash\{i\}} q_{j}(t)}{\left|\mathcal{V}_{A}(t)\right|-1} \\
\operatorname{Pr}\left(x_{i}(t+1)=\ell+\frac{\operatorname{sgn}(-\ell)}{L} \mid x_{i}(t)=\ell \in \mathcal{L}\right) \\
=\beta \operatorname{Pr}\left(\left\lfloor x_{J_{i}(t)}(t)\right\rceil=0\right)=\beta \frac{\sum_{j \in \mathcal{V}_{A}(t) \backslash\{i\}} 1-q_{j}(t)}{\left|\mathcal{V}_{A}(t)\right|-1} \\
\operatorname{Pr}\left(x_{i}(t+1)=\mathrm{NA} \mid x_{i}(t) \in \mathcal{L}\right)=\delta \\
\operatorname{Pr}\left(x_{i}(t+1)=x_{i}(t) \mid x_{i}(t) \in \mathcal{L}\right)=1-\beta-\delta \\
\operatorname{Pr}\left(x_{i}(t+1)=\ell \mid x_{i}(t)=\mathrm{NA}\right)=\frac{\gamma}{L+1} \\
\operatorname{Pr}\left(x_{i}(t+1)=\mathrm{NA} \mid x_{i}(t)=\mathrm{NA}\right)=1-\gamma
\end{array}\right.
$$

Remark 1: In the framework of opinion dynamics, the first two equations in (12) express that any individual in the social network (active agent) updates its state according to the update rule (4) with probability $\beta$. The third equation formalize the fact that individuals of the network can leave it at time $t$ (i.e., any active agent at time $t$ becomes inactive at time $t+1$ ) with probability $\delta$. The fourth equation describe the possibility of one individual in the network to not interact and preserve its opinion at time $t$ (i.e., an active agent preserves its state) with probability $1-\delta-\beta$. The last two equations express the possibility of individuals outside network to join it (choosing the state according to (7)) or stay inactive, respectively.

We can see from (12) that $x(t)$ is a Markov process as the transition probabilities only depend on its current state. However, note that $x(t) \in \mathcal{L}_{E}^{N}$. Therefore, even when $L=1$, the Markov chain has $3^{N}$ states, and $5^{N}$ states when $L=3$. In order to find the stationary state distribution of such a process would involve finding the eigenvalues of an $(L+2)^{N} \times(L+2)^{N}$ matrix which is infeasible for large $N$ (even $N>10$ ). Indeed, many modern networks have a huge number of agents in practice. These two reasons motivate us to analyze the system in the large scale limit, i.e., when $N \rightarrow \infty$.

The main objective of this work is to analyze the behavior of dynamics (12) and show that a majority of active agents in the network will reach a consensus in their action almost surely. That is, by implementing the proposed algorithm, we obtain that $\exists \epsilon^{*}>0$ s.t. $\forall \epsilon \in\left(0, \epsilon^{*}\right)$,

$$
\left\{\begin{array}{c}
\operatorname{Pr}\left(p_{+}(t+1)<\epsilon \mid p_{+}(t)<\epsilon\right)=1 \\
\operatorname{Pr}\left(p_{-}(t+1)<\epsilon \mid p_{-}(t)<\epsilon\right)=1
\end{array}\right.
$$

\section{LARGE SCALE NETWORK ANALYSIS}

For convenience, we define $p:=\left(p_{l_{0}}, p_{l_{1}}, \ldots, p_{l_{L}}\right)^{T}$ as the population vector. This allows us to define the following.
Definition 2 (Equilibrium in population): We say that $\left(p_{A}^{*}, p^{*}\right)$ is an equilibrium in population for dynamics (12) if $\exists t^{\prime} \geq 0$ such that $\left(p_{A}(t), p(t)\right)=\left(p_{A}^{*}, p^{*}\right)$ for all $t>t^{\prime}$.

Note that the $p_{A}^{*}$ denotes the population fraction of active agents and $p^{*}$ describes the distribution of the active population among the states in $\mathcal{L}$ with $\sum_{\ell \in \mathcal{L}} p_{\ell}=1$. Therefore both $p_{A}^{*}$ and $p^{*}$ are required to describe the configuration of the system in terms of population. Furthermore, $\left(p_{A}^{*}, p^{*}\right)$ is almost surely stable for the overall system if $p_{A}^{*}$ and $p^{*}$ are respectively almost surely stable for the dynamics $p_{A}(t)$ and $p(t)$.

Proposition 1: When $N \rightarrow \infty$, we have

$$
p_{A}(t+1)=(1-\delta) p_{A}(t)+\gamma\left(1-p_{A}(t)\right)
$$

almost surely, which results in an almost surely globally exponentially stable (GES) equilibrium at $p_{A}^{*}=\frac{\gamma}{\gamma+\delta}$.

Proof: Since the activation and deactivation probabilities are given by $\gamma$ and $\delta$ respectively, and these probabilities are independent of the actions/preferences, we can always write

$$
\operatorname{Pr}\left(x_{n}(t+1) \in \mathcal{L}\right)=(1-\delta) \mathbf{1}_{\mathcal{L}}\left(x_{n}(t)\right)+\gamma \mathbf{1}_{\{\mathrm{NA}\}}\left(x_{n}(t)\right)
$$

for any $n \in \mathcal{V}$ based on (12), resulting in

$$
\begin{array}{r}
\mathbb{E}\left[\sum_{n=1}^{N} \mathbf{1}_{\mathcal{L}}\left(x_{n}(t+1)\right)\right]=(1-\delta) \sum_{n=1}^{N} \mathbf{1}_{\mathcal{L}}\left(x_{n}(t)\right) \\
+\gamma \sum_{n=1}^{N} \mathbf{1}_{\{\mathrm{NA}\}}\left(x_{n}(t)\right) \\
=N\left((1-\delta) p_{A}(t)+\gamma\left(1-p_{A}(t)\right)\right)
\end{array}
$$

Note that the random variable $\mathbf{1}_{\mathcal{L}}\left(x_{n}(t+1)\right)$ has a variance greater than 0 and less than 1 . Since $N \rightarrow \infty$, we can use the central limit theorem [17] to conclude that the sampled average $p_{A}(t+1)$ will converge almost surely to the expectation, i.e., $(1-\delta) p_{A}(t)+\gamma\left(1-p_{A}(t)\right)$. The dynamics (14) can be rewritten as

$$
p_{A}^{\prime}(t+1)-p_{A}^{\prime}(t)=-(\gamma+\delta) p_{A}^{\prime}(t)
$$

where $p_{A}^{\prime}(t):=p_{A}(t)-\frac{\gamma}{\gamma+\delta}$. Since $\gamma+\delta \in(0,2)$, the origin is GES for the deterministic dynamics (17) implying $\frac{\gamma}{\gamma+\delta}$ is GES for (14). However, as (14) only occurs almost surely, the equilibrium $\frac{\gamma}{\gamma+\delta}$ is almost surely stable for the $p_{A}$ dynamics.

This result allows us to characterize the population of active agents while at equilibrium. Since this equilibrium is independent of the fraction of agents with specific preference or action states, any equilibrium of the system will have $p_{A}^{*}=\frac{\gamma}{\gamma+\delta}$. We characterize the dynamics of $p_{\ell}$, for all $\ell \in \mathcal{L}$ under this equilibrium as follows.

In the following, we consider that $\gamma$ and $\delta$ depend on $N$. We recall that $N$ is fixed but arbitrarily large and our analysis is based on the fact that large scale networks behavior is approximated by $N \rightarrow \infty$. Therefore, we will add supplementary assumptions on the behavior of $\gamma$ and $\delta$ when $N \rightarrow \infty$.

Proposition 2: If $N \rightarrow \infty$ and $\delta=O(\gamma)$, then when $p_{A}(t)=p_{A}^{*}=\frac{\gamma}{\gamma+\delta}$ (equilibrium of active agent population), we have 


$$
\begin{aligned}
p_{l_{0}}(t+1)= & (1-\delta) p_{l_{0}}(t)-\beta p_{l_{0}}(t) p_{+}(t)+\frac{\delta}{L+1} \\
& +\beta p_{l_{1}}(t) p_{-}(t) \\
p_{l_{k}}(t+1)= & (1-\beta-\delta) p_{l_{k}}(t)+\beta p_{l_{k-1}}(t) p_{+}(t)+\frac{\delta}{L+1} \\
& +\beta p_{l_{k+1}}(t) p_{-}(t) \\
p_{l_{L}}(t+1)= & (1-\delta) p_{l_{L}}(t)-\beta p_{l_{L}}(t) p_{-}(t)+\frac{\delta}{L+1} \\
& +\beta p_{l_{L-1}}(t) p_{+}(t)
\end{aligned}
$$

for all $k \in\{1, \ldots, L-1\}$ almost surely.

Proof: Let us suppose that the number of active agents is at equilibrium i.e., $p_{A}(t)=\frac{\gamma}{\gamma+\delta}$. As we assume that $\delta=$ $O(\gamma)$ one has that $\lim _{N \rightarrow \infty} \frac{\sum_{j \in \mathcal{V}_{A}(t) \backslash\{i\}} q_{j}(t)}{\left|\mathcal{V}_{A}(t)\right|-1}=p_{+}(t)$ and we can write

$$
\begin{aligned}
& \lim _{N \rightarrow \infty} \operatorname{Pr}\left(x_{n}(t+1)=l_{k}\right)=(1-\delta-\beta) \mathbf{1}_{l_{k}}\left(x_{n}(t)\right) \\
& +\beta \mathbf{1}_{l_{k-1}}\left(x_{n}(t)\right) p_{+}(t)+\frac{\gamma}{L+1} \mathbf{1}_{\mathrm{NA}}\left(x_{n}(t)\right) \\
& +\beta \mathbf{1}_{l_{k+1}}\left(x_{n}(t)\right) p_{-}(t)
\end{aligned}
$$

for any $k \in\{1, \ldots, L-1\}$ based on (12). Using the central limit theorem as done before in Proposition 1, we can evaluate

$$
\begin{aligned}
& \frac{\sum_{n=1}^{N} \mathbf{1}_{l_{k}}\left(x_{n}(t)\right)}{N}=(1-\beta-\delta) p_{l_{k}}(t) p_{A}(t) \\
& +\frac{\gamma}{L+1}\left(1-p_{A}(t)\right)+\beta p_{l_{k-1}}(t) p_{+}(t) p_{A}(t) \\
& +\beta p_{l_{k+1}}(t) p_{-}(t) p_{A}(t)
\end{aligned}
$$

almost surely. Dividing by $p_{A}(t)>0$ on both sides, we have the left hand side becoming $p_{l_{k}}(t+1)$ and using $\gamma \frac{1-p_{A}(t)}{p_{A}(t)}=\delta$ one obtains (18) for all $\ell \in\left\{l_{1}, \ldots, l_{L-1}\right\}$. We can similarly evaluate for $p_{l_{0}}(t+1)$ and $p_{l_{L}}(t+1)$, but by keeping in mind that $\phi\left(l_{0}, 0\right)=l_{0}$ and $\phi\left(l_{L}, 1\right)=l_{L}$.

Proposition 2 converts the stochastic dynamics of our Markovian system (12) into a deterministic population dynamics (18) that will occur with probability 1 when $N \rightarrow \infty$. Next, we characterize the equilibrium points of (18) when $\delta=O(1 / N)$ and $\gamma=O(1 / N)$.

Theorem 1: When $N \rightarrow \infty, \delta=O(1 / N), \gamma=O(1 / N)$, $\frac{N \gamma}{\gamma+\delta} \rightarrow \infty, L \geq 3$ and $p_{A}(t)=p_{A}^{*}$, the system (12) has exactly two almost surely locally exponentially stable equilibrium points at

$$
p_{S 1}^{*}=(1,0, \ldots, 0)^{T} \text { and } p_{S 2}^{*}=(0, \ldots, 0,1)^{T} .
$$

The system also allows for one unstable equilibrium point at

$$
p_{U}^{*}=\frac{1}{L+1} \cdot(1,1, \ldots 1)^{T} .
$$

Proof: See Appendix A.

From Theorem 1, we can see that taking $L \geq 3$ will result in almost all active agents with a common action at equilibrium when $\delta=O(1 / N)$ and $\gamma=O(1 / N)$. Since Theorem 1 provides local exponential stability of (18) around $p_{S 1}^{*}$, we can write

$$
\exists \epsilon^{*}>0 \text { s.t. } \forall \epsilon<\epsilon^{*}, \operatorname{Pr}\left(p_{\ell}(t+1)<\epsilon \mid p_{\ell}(t)<\epsilon\right)=1
$$

for all $\ell \in \mathcal{L} \backslash\left\{l_{0}\right\}$ which shows that the first condition of objective (13) is satisfied. Considering the other locally stable point $p_{S 2}^{*}$, we can also prove (13) is satisfied for the other condition.

Although these results are derived for $N \rightarrow \infty$, we perform simulations and show that that the probability of retaining consensus is close to 1 when $N$ is large but finite.

Remark 2 (Case of $L=1$ ): The case of $L=1$ which is the only case excluded from Theorem 1 (recall $L$ is odd) implies that $x_{n}(t)=q_{n}(t)$ as $\mathcal{L}=\{0,1\}$ and this results in a dynamics similar to an Ising model used in [18]. We observe that equation (18) can be simplified into

$$
\begin{aligned}
p_{l_{0}}(t+1) & =(1-\delta) p_{l_{0}}(t)-\beta p_{l_{0}}(t) p_{l_{L}}(t)+\beta p_{l_{L}}(t) p_{l_{0}}(t) \\
& =(1-\delta) p_{l_{0}}(t)+\delta / 2 \\
p_{l_{L}}(t+1) & =(1-\delta) p_{l_{L}}(t)-\beta p_{l_{0}}(t) p_{l_{L}}(t)+\beta p_{l_{L}}(t) p_{l_{0}}(t) \\
& =(1-\delta) p_{l_{L}}(t)+\delta / 2
\end{aligned}
$$

which is almost surely GES at $p_{U}^{*}$ and which results in $p_{+}(t)=p_{-}(t)=0.5$. Therefore taking $L=1$ ensures that there will be no consensus at equilibrium.

\section{NUMERICAL RESUltS}

In this part, we numerically illustrate the theoretical results presented in the previous Section. These simulations show that as far as the arrivals/departures occur sufficiently rarely with respect to the dimension of the network ( $N \delta$ and $N \gamma$ are small) we obtain majority action consensus.

For our simulations we take $L=3$, i.e. $\mathcal{L}=$ $\{0,1 / 3,2 / 3,1\}$ and we fix $\beta=0.5$. In our first set of simulations we plot the population of agents with action 1 , i.e., $p_{+}(t)$ vs $t$ with $\delta=\gamma=0.01$ for various values of $N$. In Figure 1a, when $N=10$, we see how consensus is sometimes perturbed by the activation of new agents. When $N$ sufficiently large as in Figure 1b, consensus is maintained for a very long duration of over $10^{5}$ time instants.

In Figure 2, we plot the population with action 1 against time for $N=1000$ and various values of $\delta=\gamma$. When $\delta=0.01$, the simulation results are close to the theoretical behavior predicted for $N \rightarrow \infty$ and $\delta=O(1 / N)$. When $\delta=0.2$, the influence of the agents who get activated or deactivated is dominant resulting in random actions, and there is no majority action preservation. Interestingly, even with $\delta=0.1$, about $75 \%$ of the agents maintain a common action over a long duration.

\section{CONClusion}

We introduced and analyzed an open multi-agent system with discrete states and quantized information. Basically, the system is split into two subsets: active agents and inactive ones. Each active agent is characterized by a state belonging to a finite set while the inactive agents are just artificially introduced to preserve the dimension of the overall system. The system dynamics is modeled as a gossiping in which the active agents access only the action (quantization of the state) of neighbours. At any time the network can be affected 


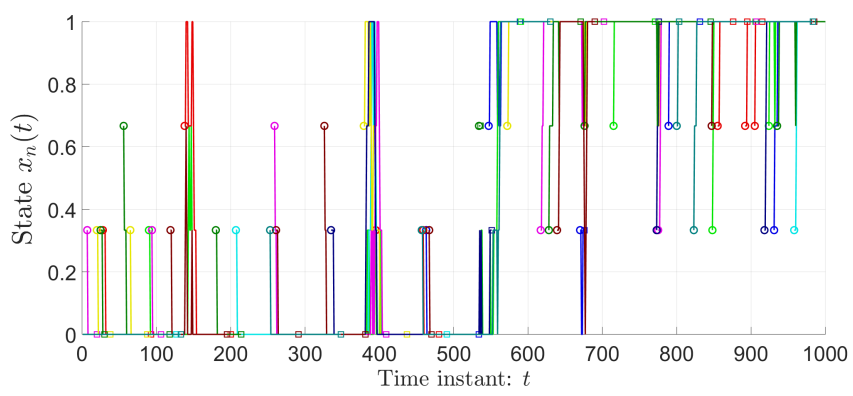

(a) Dynamics of each agent when $N=10$. Agent activation is marked by a circle and deactivation with a square. At around $t=$ 560 , the majority agreement value at 0 is perturbed to become 1 .

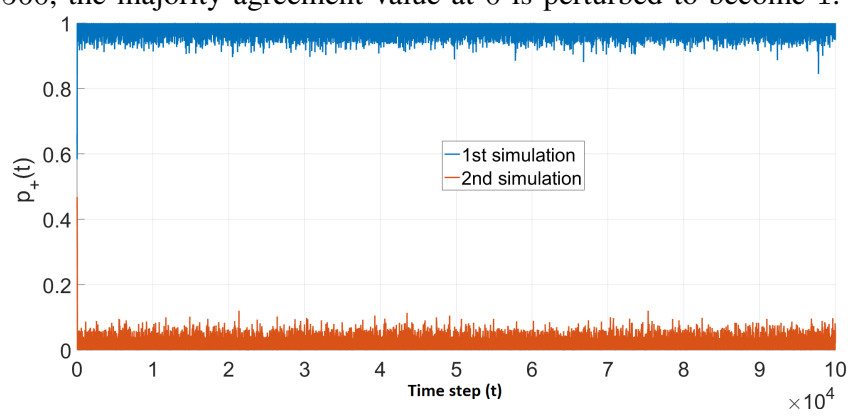

(b) Population dynamics, i.e. fraction of agents with action 1 vs time when $N=100$ and the almost surely stable equilibrium (defined when $N \rightarrow \infty$ ) persists for a very long duration (much more than $10^{5}$ steps).

Fig. 1: Agent dynamics for $\delta=0.01$.

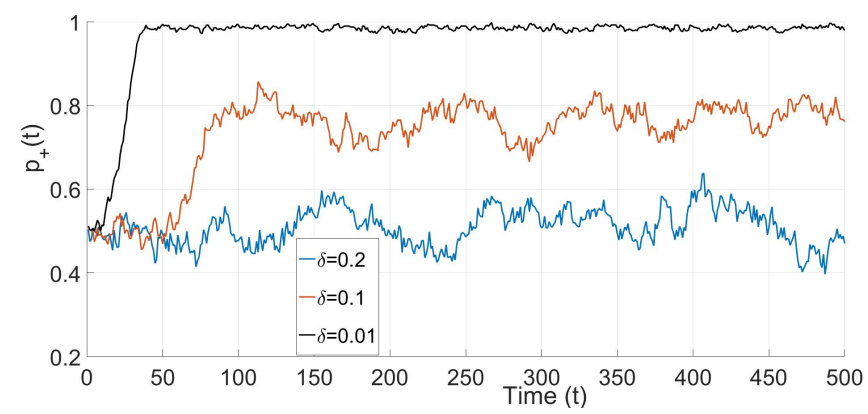

Fig. 2: Plotting $p_{+}(t)$ vs $t$ for various values of $\delta$. We observe that even when $\delta=0.1$, i.e. about 100 agents are being activated or deactivated in the network at each time instant, a majority of agents hold the same action for a long period of time.

by arrivals and departures of some agents (i.e., some active agents can become inactive and vice-versa). These events occur randomly as a Bernoulli process. We show that, in this framework, classical consensus can be replaced by a weaker formulation: a large fraction of the active population has almost surely reached a common action.

\section{APPENDIX}

\section{A. Proof of Theorem 1}

Proof: In Proposition 2 we have shown that the population dynamics associated with the Markovian system
(12) can be given by (18) almost surely. Since we have $\delta=O(1 / N)$ and $\gamma=O(1 / N)$, as $N \rightarrow \infty, \delta, \gamma \rightarrow 0$, (18) will be transformed into

$$
\begin{aligned}
p_{l_{0}}(t+1)= & p_{l_{0}}(t)-\beta p_{l_{0}}(t) p_{+}(t)+\beta p_{l_{1}}(t) p_{-}(t) \\
p_{l_{k}}(t+1)= & (1-\beta) p_{l_{k}}(t)+\beta p_{l_{k-1}}(t) p_{+}(t) \\
& +\beta p_{l_{k+1}}(t) p_{-}(t) \\
p_{l_{L}}(t+1)= & p_{l_{L}}(t)-\beta p_{l_{L}}(t) p_{-}(t)+\beta p_{l_{L-1}}(t) p_{+}(t)
\end{aligned}
$$

for $k \in\{1, \ldots, L-1\}$ at $p_{A}(t)=p_{A}^{*}$. Firstly, we can easily verify that $p_{S 1}^{*}, p_{S 2}^{*}$, and $p_{U}^{*}$ are all equilibria. At $p_{S 1}^{*}$, we have $p_{+}(t)=0$ and $p_{-}(t)=1$ which results in $p_{\ell}(t+1)=$ $p_{\ell}(t)$ for all $\ell \in \mathcal{L}$ and similar arguments hold for $p_{S 2}^{*}=1$. At $p_{U}^{*}$, we have $p_{+}(t)=p_{-}(t)=0.5$ which once again results in an equilibrium.

For the stability analysis of (23), we perform a linear analysis of the dynamics around these equilibria by studying the Jacobian matrix. Denote by $g_{k}(\mathbf{p})$ the dynamics of the population with preference state $l_{k} \in \mathcal{L}$, i.e. $p_{l_{k}}(t+1)=$ $g_{k}(\mathbf{p})$. For the rest of the proof, for ease of exposition, we omit the argument $t$ from $p_{\ell}(t)$.

If we denote the Jacobian elements by $J_{i, j}$, where $J_{i, j}=$ $\frac{\partial g_{i}}{\partial p_{l_{j}}}$, then for all $0<i \leq \frac{L-1}{2}$, and for all $\frac{L+1}{2} \leq j<L$, we have:

$$
\begin{aligned}
& J_{0,0}=1+\beta p_{l_{1}}-\beta\left(\sum_{k=(L+1) / 2}^{L} p_{l_{k}}\right) \\
& J_{i, i}=1-\beta+\beta p_{l_{i+1}} \\
& J_{j, j}=1-\beta+\beta p_{l_{j-1}} \\
& J_{L, L}=1+\beta p_{l_{L-1}}-\beta\left(\sum_{k=0}^{(L-1) / 2} p_{l_{k}}\right)
\end{aligned}
$$

We also have ,

$$
\begin{gathered}
\forall i \in\{2, \ldots,(L-1) / 2\}, \quad J_{0, i}=-\beta p_{l_{0}} \\
\forall i \in\{(L+1) / 2, \ldots, L-2\}, \quad J_{0, i}=\beta p_{l_{1}}
\end{gathered}
$$

and

$$
\begin{gathered}
\forall i \in\{2, \ldots,(L-1) / 2\}, \quad J_{L, i}=\beta p_{l_{L-1}} \\
\forall i \in\{(L+1) / 2, \ldots, L-2\}, \quad J_{L, i}=-\beta p_{l_{L}} .
\end{gathered}
$$

For all $i, j \in\{1,2, \ldots, L-1\}$ such that $|i-j|>1$ we have

$$
J_{i, j}=\beta p_{l_{i+1}}
$$

when $j \leq(L-1) / 2$ and

$$
J_{i, j}=\beta p_{l_{i-1}}
$$

when $j \geq(L+1) / 2$. Next, we have

$$
J_{0,1}=\beta\left(p_{l_{1}}+\sum_{k=0}^{(L-1) / 2} p_{l_{k}}\right)
$$

$$
J_{L, L-1}=\beta\left(p_{l_{L-1}}+\sum_{k=(L+1) / 2}^{L} p_{l_{k}}\right) \text {. }
$$


For all $i \in\{1,2, \ldots, L-1\}$, we have

$$
J_{i, i+1}=\beta p_{l_{k}}+\beta\left(\sum_{j=0}^{(L-1) / 2} p_{l_{j}}\right)
$$

where $k=i+1$ if $i+1 \leq(L-1) / 2$ and $k=i-1$ otherwise; and

$$
J_{i, i-1}=\beta p_{l_{k}}+\beta\left(\sum_{j=(L+1) / 2}^{L} p_{l_{j}}\right)
$$

where $k=i+1$ if $i-1 \leq(L-1) / 2$ and $k=i-1$ otherwise.

We can study the stability of an equilibrium point by looking at the eigenvalues of Jacobian matrix evaluated at the equilibrium points, i.e.,

$$
J\left(p_{S 1}^{*}\right)=\left[\begin{array}{llll}
1 & \beta & 0 & \cdots \\
0 & 1-\beta & \beta & \cdots \\
0 & 0 & 1-\beta & \cdots \\
\vdots & & &
\end{array}\right]
$$

which is a triangular matrix. This matrix therefore has the eigenvalues 1 and $1-\beta$. This can be easily verified by evaluating the determinant of the $\mathbf{J}\left(p_{S_{1}}^{*}\right)-\lambda \mathbf{I}$. The eigenvector corresponding to the eigenvalue of 1 is in fact $(1,0, \ldots)$. Since we are working on the unit simplex (as $\sum_{\ell \in \mathcal{L}} p_{\ell}=1$ ), the equilibrium $p_{S 1}^{*}$ is locally exponentially stable within the unit simplex. By symmetry, we can also show the same for the equilibrium $p_{S 2}^{*}$. However, the dynamics (23) occurs almost surely and is not deterministic, which results in these two equilibria being almost surely stable.

Now, we study the Jacobian at the other equilibrium $p_{U}^{*}$. Here, $p_{+}=p_{-}=0.5$ and the $J$ at this point can be evaluated. Its first column $\left(J_{0,0}, J_{1,0}, \ldots, J_{L, 0}\right)^{T}$ is given by

$$
\left(1+\frac{\beta}{L+1}-\frac{\beta}{2}, \frac{\beta}{L+1}+\frac{\beta}{2}, \frac{\beta}{L+1}, \ldots, \frac{\beta}{L+1},-\frac{\beta}{L+1}\right)^{T}
$$

The columns $j$ for $j \in\{1, \ldots,(L-1) / 2\}$ are of the form

$$
\begin{array}{r}
\left(\frac{\beta}{L+1}, \ldots, 1+\frac{\beta}{L+1}+\frac{\beta}{2}, \frac{\beta}{L+1}-\beta, \frac{\beta}{L+1}+\frac{\beta}{2}\right. \\
\left., \ldots, \frac{\beta}{L+1},-\frac{\beta}{L+1}\right)^{T}
\end{array}
$$

where $1+\frac{\beta}{L+1}+\frac{\beta}{2}$ is the diagonal term of the Jacobian. The $\mathrm{j}$-th column for $j \in\{(L+1) / 2, \ldots, L-1\}$ is of the form

$$
\begin{array}{r}
\left(-\frac{\beta}{L+1}, \frac{\beta}{L+1}, \ldots, 1+\frac{\beta}{L+1}+\frac{\beta}{2}, \frac{\beta}{L+1}-\beta\right. \\
\left., \frac{\beta}{L+1}+\frac{\beta}{2}, \ldots, \frac{\beta}{L+1}\right)^{T}
\end{array}
$$

Finally, the $L$-th column is given by

$$
\left(-\frac{\beta}{L+1}, \frac{\beta}{L+1}, \ldots, \frac{\beta}{L+1}, \frac{\beta}{L+1}+\frac{\beta}{2}, 1+\frac{\beta}{L+1}-\frac{\beta}{2}\right)^{T}
$$

The above matrix is such that each column has exactly one element which is $-\frac{\beta}{L+1}$ either at the first row (after column index is more than $(L-1) / 2$ ) or at the last row. We can evaluate the sum of each column as

$$
\sum_{i=0}^{L} J_{i, j}\left(p_{U}^{*}\right)=1+(L-2) \frac{\beta}{L+1},
$$

when $L \geq 3$ for all $j \in\{0,1, \ldots, L\}$. We can show that one of the eigenvalues of the matrix $J\left(p_{U}^{*}\right)$ is $1+\frac{\beta}{L+1}(L-2)$. To do this, we look at

$$
\operatorname{det}\left(J\left(p_{U}^{*}\right)-\left(1+(L-2) \frac{\beta}{L+1}\right) I_{L+1}\right)
$$

Using the property that adding a scalar times a row to another row does not change the determinant, we replace the first row with the sum of all rows, and this results in the first row becoming all zeroes since we know from (24) that the sum of each column is 0 by adding $-1-(L-2) \frac{\beta}{L+1}$ once to each row. Since $J\left(p_{U}^{*}\right)$ has an eigenvalue $1+(L-2) \frac{\beta}{L+1}>1$ when $L \geq 3$, we see that $p_{U}^{*}$ is unstable when $L \geq 3$.

\section{REFERENCES}

[1] F. Bullo, J. Cortés, and S. Martinez, Distributed Control of Robotic Networks. A Mathematical Approach to Motion Coordination Algorithms. Princeton University Press, 2009.

[2] M. Mesbahi and M. Egerstedt, Graph theoretic methods in multiagent networks. Princeton University Press,Princeton. NY, 2010.

[3] J. Chow and P. Kokotović, "Time scale modeling of sparse dynamic networks," IEEE Transactions on Automatic Control, , vol. 30, no. 8 , pp. 714-722, 1985.

[4] E. Bıyık and M. Arcak, "Area aggregation and time-scale modeling for sparse nonlinear networks," Systems \& Control Letters, vol. 57, no. 2, pp. 142-149, 2007.

[5] R. Hegselmann and U. Krause, "Opinion dynamics and bounded confidence models, analysis, and simulation," Journal of Artificial Societies and Social Simulation, vol. 5, no. 3, 2002.

[6] N. E. Friedkin and E. C. Johnsen, "Social influence and opinions," Journal of Mathematical Sociology, vol. 15, no. 3-4, pp. 193-206, 1990.

[7] A. Jadbabaie, J. Lin, and A. S. Morse, "Coordination of groups of mobile autonomous agents using nearest neighbor rules," IEEE Transactions on Automatic Control,, vol. 48, no. 6, pp. 988-1001, 2003.

[8] J. M. Hendrickx and J. N. Tsitsiklis, "Convergence of type-symmetric and cut-balanced consensus seeking systems." IEEE Transactions on Automatic Control, vol. 58, no. 1, pp. 214-218, 2013.

[9] L. Moreau, "Stability of multiagent systems with time-dependent communication links," IEEE Transactions on Automatic Control, vol. 50, no. 2, pp. 169-182, 2005.

[10] J. Török, G. Iniguez, T. Yasseri, M. S. Miguel, K. Kaski, and J. Kertész, "Opinions, conflicts, and consensus: modeling social dynamics in a collaborative environment," Physical review letters, vol. 110, no. 8, p. 088701, 2013.

[11] G. Iniguez, J. Török, T. Yasseri, K. Kaski, and J. Kertész, "Modeling social dynamics in a collaborative environment," EPJ Data Science, vol. 3, no. 1, 2014.

[12] C. Carrascosa, A. Giret, V. Julian, M. Rebollo, E. Argente, V. B. . I. F for Autonomous Agents, and . Multiagent Systems, "Service oriented mas: an open architecture." in In Proceedings of The 8th International Conference on Autonomous Agents and Multiagent Systems, vol. 2, 2009, pp. $1291-1292$.

[13] J. Hendrickx and S. Martin, "Open multi-agent systems: Gossiping with deterministic arrivals and departures." in 54th Annual Allerton Conference on Communication, Control, and Computing, 2016.

[14] V. Varma and I. Morărescu, "Modeling stochastic dynamics of agents with multi-leveled opinions and binary actions," in Proc. of the 56th IEEE Conference on Decision and Control, 2017.

[15] P. Frasca, R. Carli, F. Fagnani, and S. Zampieri, "Average consensus on networks with quantized communication," International Journal of Robust and Nonlinear Control, vol. 19, no. 16, pp. 1787-1816, 2009.

[16] A. C. Martins, "Discrete opinion models as a limit case of the coda model," Physica A: Statistical Mechanics and its Applications, vol. 395, pp. 352-357, 2014

[17] P. Billingsley, Convergence of probability measures. John Wiley \& Sons, 2013.

[18] E. Ising, "Contribution to the theory of ferromagnetism," Ph.D. dissertation, University of Hamburg, 1924 


\section{University Library}

\section{- $\mathrm{M}$ IIN E R VA A gateway to Melbourne's research publications}

Minerva Access is the Institutional Repository of The University of Melbourne

Author/s:

Varma, VS;Morarescu, IC;Nesic, D

Title:

Open Multi-Agent Systems with Discrete States and Stochastic Interactions

Date:

2018-07-01

Citation:

Varma, V. S., Morarescu, I. C. \& Nesic, D. (2018). Open Multi-Agent Systems with Discrete States and Stochastic Interactions. IEEE Control Systems Letters, 2 (3), pp.375-380. https:// doi.org/10.1109/LCSYS.2018.2840431.

Persistent Link:

http://hdl.handle.net/11343/251759 